ringste Menge eines zuckerartigen Körpers abzuscheiden, obwohl diese Behandlungen ohne Zweifel wesentliche Veränderungen des Gerbstoffes im Gefolge hatten. Es entstehen bei den betreffenden Operationen wohl immer Substanzen, die Fehling'sche Lösung reduciren, die aber durch Bleiessig fällbar sind. Auch mittels Phenylhydrazin war aus der Kaffeegerbsäure kein Zucker, bez. kein Osazon zu erhalten.

Ich kann also hinsichtlich des Zuckers des Kaffeegerbstoffes keine der bisherigen Angaben bestätigen, muss vielmehr aus meinen Beobachtungen den Schluss ziehen, dass die fragliche Gerbsäure überhaupt keinen Zucker enthält und mithin kein Glycosid ist.

Die Untersuchung der Kaffeegerbsäure wird von mir fortgesetzt; ich behalte mir weitere Mittheilungen vor.

\section{Ueber den \\ Einfluss der Wasserstoffionenconcentration bei der Einwirkung der Halogenate, speciell des Jodats, auf die Halogenide. ${ }^{1}$ ) Von Hugo Ditz und B. M. Margosches.}

Die Halogene Chlor, Brom und Jod, derselben Gruppe des periodischen Systems angehörend, zeigen in ihrem chemischen Verhalten vielfach eine stufenweise Regelmässigkeit, entsprechend der Höhe ihrer Atomg(wichte. So nimmt auch die Wärmeentwicklung bei der Bildung der verdünnten Halogenwasserstoffsäuren mit zunehmendem Atomg(:wichte regelmässig ab; entsprechend der Abnahme des Energieunterschiedes zwischen dem freien Elemente und seinem Ion erfolgt eine Verminderung der Ionisirungstendenz mit steigendem Atomgewichte. Letztere zeigt sich z. B. bei der Einwirkung des Halogens mit niedrigerem Atomgewichte auf ein $\mathrm{Salz}$ der Wasserstoffsäure des Halogens mit höherem Atomgewichte, wobei das Ion des letzteren in den elementaren Zustand übergeht, während jenes sich in das Ion verwandelt.

Die Abnahme der Ionisirungstendenz mit steigendem Atomgewichte tritt nun auch in dem Verhalten der Halogenwasserstoffsäuren bez. deren Salze gegenüber Oxydation:smitteln hervor. Besonderes Interesse beanspruchen hier die Fälle, in welchen die letzteren durch die verschiedenen Halogensauerstoffsäuren bez. deren Salze vertreten sind, schon aus dem Grunde, da die hierbei

1) Unter Halogeniden verstehen wir im ganzen Verlanfe der Abhandlung Chlorid, Bromid und Jodid, schliessen also Fluorid aus. in Betracht kommenden Reactionen für die analytische Chemie vielfach von Bedeutung sind. Wiewohl einzelne dieser Reactionen in ihrem Verlaufe schon einem gründlicheren Studium unterzogen worden $\sin ^{3}$ ), ist eine allgemeine Betrachtung des bei den verschiedenen Processen stattfindenden Reactionsverlaufes und der hierbei maassgebenden Factoren ron einem einheitlichen Gesichtspunkte aus unseres Wissens noch nicht erfolgt und daher wohl am Platze.

Die analytischen Reactionen der Anionen der Halogensauerstoffsäuren bez. deren Salze sind bekanntlich keine eigentlichen Ionenreactionen, sondern bestehen in der Mehrzahl der Fälle in der leichten Sauerstoffabgabe derselben. Zum Unterschiede von den Sanerstoffsäuren vom Typus $\mathrm{HXO}_{3}$ sind die niederen Sauerstoffsäuren, speciell die des Chlors und Broms ausserordentlich schwache Säuren. Die Ursache dieser Erscheinung kann nach Ostwald ${ }^{3}$ ) am ehesten in einem Wechsel der Valenz des Halogens gesucht werden, steht aber im auffallenden Gegensatze zu der sonstigen acidificirenden Wirkung des Sauerstoffes. Charakteristisch für die niederen Sauerstoffsäuren der Halogene, speciell für die unterchlorige und unterbromige Säure, ist der rasche Oxydationsverlauf beim Zusammenbringen ihrer Salze mit den verschiedenen Halogenwasserstoffsüuren, der, ohne dass ein Überschuss an Säure erforderlich ist, sofort verläuft. Betrachten wir z. B. die Verhältnisse, wie sie bei der unterchlorigen Säure zum Unterschiede von der Chlorsäure bestehen, so ist schon aus den berechneten Wärmemengen, welche bei der Oxydation durch die beiden Säuren frei werden, zu ersehen, dass die Oxydationsfähigkeit der Chlorsäure geringer ist als die der unterchlorigen Säure. $\mathrm{Ob}$ die dementsprechend grössere Reactionsgeschwindigkeit bei der durch unterchlorige Säure bewirkten Oxydation die alleinige Ursache für den im Vergleiche zur Chlorsäure verschiedenen Oxydationsverlauf bildet, ist nicht klargestellt $t^{4}$ ).

2) Da diese Abhandlung mehr den Charakter einer vorläufigen Mittheilung trägt, haben wir von der vollständigen Zusammenstellung und Angabe der einschlägigen Litteratur abgesehen. Wir wollen hier nur erwähnen: O. Burchhard, Über die Oxydation der Jodwasserstoffsäure durch die Sauerstoffsäuren der Halogene. Inaug.- Dissert. Tübingen (nach Chem.-Ztg. 1888, 1750). H. Schlundt, Über die Schnelligkeit der Jodausscheidung in gemischten Lösungen von Kaliumchlorat, Jodkalium und Salzsäure (Chem.-Ztg. Rep. 1896, 21).

3) Wissenschaftliche Grundlagen der analytischen Chemie, 3. Aufl. 1901, 182.

4) Vielleicht spielt hierbei auch der Umstand eine Rolle, dass die niederen Sauerstoffsäuren der 
Die Halogensauerstoffsäuren vom Typus $\mathrm{HXO}_{3}$ sind als starke Säuren in ihren wässerigen Lösungen weitgehend dissociirt. Das Verhalten der einzelnen Halogensauerstoffsäuren bez. deren Salze zu den verschiedenen Halogeniden ist nun in erster Linie abhängig von der Ionisirungstendenz der betreffenden Halogene. Ein und dasselbe Oxydationsmittel, also in unserem Falle ein Halogenat, wird, unter sonst gleichen Umständen (Menge der vorhandenen Säure, Concentration u. s. w.) auf die verschiedenen Halogenide zur Einwirkung gebracht, die Überführung des Ions in den elementaren Zustand im Allgemeinen am leichtesten bei. den Jodiden, schwerer bei den Bromiden und am schwersten bei den Chloriden durchführen.

Nach diesen allgemeinen Bemerkungen gehen wir speciell zur Betrachtung des Verhaltens von Jodat zu den Halogeniden über. Versetzt man eine Lösung von Kaliumjodat mit einem Überschusse von Kaliumjodid und bringt nun durch Zusatz von verdünnter Salz- oder Schwefelsäure Wasserstoffionen zu der Lösung, so erfolgt bekanntlich der Reactionsverlauf momentan und vollständig entsprechend der Gleichung:

$\mathrm{KJO}_{3}+5 \mathrm{KJ}+6 \mathrm{HCl}=3 \mathrm{~J}_{2}+6 \mathrm{KCl}+3 \mathrm{H}_{2} \mathrm{O}$.

Zum vollständigen Verlaufe dieser Reaction genügt es, die theoretisch nothwendige, der Gleichung entsprechende Säuremenge zuzusetzen, wodurch auch, wie bekannt, neben der Anwendung des Kaliumjodats als Urtitersubstanz für die Gehaltsbestimmung von Natriumthiosulfatlösungen, die Bestimmung geringer Säuremengen auf jodometrischem Wege ermöglicht ist ${ }^{5}$ ).

Wird die Reduction des Jodats nun nicht durch Jodid, sondern durch Bromid (bei Gegenwart von Wasserstoffionen) vorgenommen, so ist der Reactionsverlauf ein keineswegs so glatter. Nach S. Bugarsaky ${ }^{6}$ )

Halogene sehr schwachen Säurecharakter besitzen, d. h. in wässeriger lösung fast vollständig im nichtdissociirten Zustande vorhanden sind und dadurch möglicherweise eine entsprechend grössere Oxydationswirkung verursachen. Dafür spricht auch die Thatsache, dass die freie, nichtdissociirte unterchlorige Säure stärker oxydirend wirkt als das Unterchlorigsüure-Anion der unterchlorigsauren Salze. Letzteres wird auch durch Einwirkung der freien unterchlorigen Säure nach Foerster und Jorre (Journ. f. prakt. Chem. 1899, 59, 84) in Chloration übergeführt nach der Gleichung:

$$
2 \mathrm{HOCl}+\overrightarrow{\mathrm{ClO}}=2 \overrightarrow{\mathrm{H}}+2 \overrightarrow{\mathrm{Cl}}+\mathrm{ClO}_{3} \text {. }
$$

(Siehe diesbezüglich auch die Fussnote bei Chlorat.)

5) Siehe diesbezüglich auch M. Gröger (Zeitschr. f. angew. Chemie 1890, 363) u. F. Fessel (Zeitschr. f. anorg. Chemie 1900, 23, 66).

6) Zeitschr. f. anorg. Chem. 1896, 10, 387. Bugarszky basirte seime Arbeit auf die Messungen verläuft die Reaction zwischen Jodsäure und Bromwasserstoff sehr langsam, so dass selbst bei Anwendung von concentrirteren Lösungen das Brom nicht quantitativ erhalten wird, wenn man die Reaction bei gewöhnlicher Temperatur vor sich gehen lässt und das nach der Gleichung

$$
\begin{aligned}
& 2 \mathrm{KJO}_{3}+10 \mathrm{KBr}+6 \mathrm{H}_{2} \mathrm{SO}_{4} \\
= & 5 \mathrm{Br}_{2}+\mathrm{J}_{2}+6 \mathrm{~K}_{2} \mathrm{SO}_{4}+6 \mathrm{H}_{2} \mathrm{O}
\end{aligned}
$$

ausgeschiedene Brom und Jod mit Chloroform oder Schwefelkohlenstoff ausschüttelt. Bugarszky nimmt daher die Zersetzung in der Wärme vor ind gelingt es ihm, das im Auge gehabte Ziel, die Ausarbeitung einer Methode zur quantitativen Trennung von Brom und Chlor, zu erreichen, indem bei Anwendung bestimmter Säuremengen und Einhaltung entsprechender Concentrationsverhältnisse der Reactionsverlauf ein quantitativer ist, ohne dass gleichzeitig vorhandenes Chlorid durch den Überschuss der 'Jodsäure oxydirt wird. Zum quantitativen Verlaufe der zuletzt angegebenen Reaction ist neben der Wärmezufuhr zum Unterschiede von der bei Gegenwart von Jodid stattfindenden Reduction ein gewisser Säureüberschuss erforderlich. Es spielt also hier neben der Ionisirungstendenz ein zweiter Factor eine Rolle, es ist dies die Menge der vorhandenen Säure bez. die Concentration der Wasserstoffionen. Wie des Weiteren zu ersehen ist, steht der Einfluss der letzteren in einem gewissen Zusammenhange mit der Ionisirungstendenz, indem bei Zunahme dieser unter sonst gleichbleibenden Verhältnissen (also auch bei gleichem Oxydationsmittel) die Menge der Wasserstoffionen, welche erforderlich ist, um den Übergang des betreffenden Ions in den elementaren $\mathrm{Zu}$ stand unter gleichzeitiger Reduction des Jodats herbeizuführen, wächst.

Um speciell den Einfluss der Wasserstoffionenconcentration bei der Einwirkung von Jodat- auf Jodionen zum Unterschiede von der auf Bromionen zu untersuchen, stellten wir einige Versuche an:

Versetzt man eine Jodat und Jodid enthaltende Lösung auch nur mit einem Tropfen einer $1 / 10 \mathrm{~N}$.-Salz-oder Schwefelsäure, so zeigt der Eintritt der Jodstärkereaction schon den theilweisen Übergang des Jodions in den elementaren Zustand. Wird hingegen eine Jodat und Bromid enthaltende Lösung mit der gleichen Menge $1 / 10$ N.-Säure versetzt, so tritt keine Veränderung ein und findet selbst bei Zusatz einer grösseren Anzahl von ccm 1/10 N.-Säure (je nach der Menge und Con-

Bancroft's über die elektromotorische Kraft der Reductions- und Oxydationsketten (Zeitschr. f. physik. Chemie 1892, 10, 387). 
centration des in der Lösung vorhandenen Salzgemisches) die Reduction nicht statt, indem die so behandelte Lösung zugesetzt'm Schwefelkohlenstoff (oder Tetrachlorkohlenstoff) nicht die geringste Färbung verleiht. So konnten bei Anwendung von 25 crm $1 / 10 \mathrm{~N} .-\mathrm{KJO}-$ und $10 \mathrm{~cm}$ 1/10 N.-KBr-Lösung $15 \mathrm{ccm}$ einer ungefähr $1 / 2 \mathrm{~N}$.-HCl zugegeben werden, bis beigefügter Schwefelkohlenstoff das Auftreten der freien Halogene anzeigte. Bei einem zweiten, mit gleichen Mengen der Salzlösungen ausgeführten Versuch war der Beginn der Reaction bei Zusatz von $250 \mathrm{cim}$ 1/10 N.-Säure noch nicht wahrzunehmen. Iiei zunehmender Terdünnung ist also eine hedeutend grössere Säuremenge erforderlich, um den Beginn der Reaction hervorzurufen ${ }^{7}$ ). Während also bei Anwendung von. Kaliumjodat als Oxydationsmittel schon geringe Mengen Wasserstoffionen im Stande sind, gleichzeitig anwesendes Jodion in den elementaren Zustand überzuführen, ist ein bestimmtes Minimum an Wasserstoffionen nothwendig, um den Oxydationsprocess bei Anwesenheit von Bromid einzuleiten. Dass nicht nur der Eintritt der Reaction, sondern auch nawh diesem der vollständige Verlauf derselben an das Vorhandensein überschüssiger Wassurstoffionen geknüpft ist, zeigte ein Versurh, bei dem, nachdein die zur Einleitung der Reaction nothwendige Säure zugesetzt worden war, noch die der Reactionsgleiclung entsprechende Menge an 1/10 N.-Säure himzugegeben wurde. Wie vorauszusehen war, ergab sich schon beim Vergleiche der Intensität der Färbung mit einem bei grösser m Säureüberschusse ausgeführten Versuche, diss die Reaction in ersterem Falle nur zum geringsten Theile vor sich ging, das Freiwerden von Brom aber mit der Zeit zunahm. Dass bei der Einwirkung ron Kaliumjodat suf Chlorid die anzuwendende Säuremenge behufs Einleitung der Reaction eine noch beträchtlich grössere als bei Bromid sein muss, ist schon aus der Arbeit von Bugarszky zu ersehen, da ja, wie erwähnt, bei den von ihm eingehaltenen Umständen Bromide neben Chloriden bestimmt werden können, letztore also nicht Oxydation erfahren.

Der Einfluss der Wasserstoffionenconcentration bei der Einwirkung von Jodat auf die drei Halogenide liess es möglich erscheinen, durch successiven Zusatz von verdünnter Sïure auf

7) Wenn anch die absolute Menge der Wasserstoffionen bei gleicher Sïuremenge mit steigeniler Verdünnung zunimmt, so ist die relative Menge der Wasserstoffionen im Verhältniss zu den in ter Lösung vorhandenen Salzen bei der concentrirteren Flüssigkeit in der Volumeneinheit grösstry, in latzterem Falle also die Concentration der Wasserstoffionen eine grössere. die mit überschüssigem Jodat versetzte I_ösung ron Jodid und Bromid bez. Chlorid das Jodion in elementares Jod überzuführen, ohne dass das Bromid oder Chlorid dabei einen Angriff erfährt.

Einige Torversuche wurden in der Weise vorgenommen, dass eine bestimmte Menge einer Kaliumjodidlösung mit einem bestimmten Überschusse von Kaliumjodatlösung versetzt, successive $z u$ je 2 bis $3 \mathrm{ccm} 1 / 10 \mathrm{~N} .-\mathrm{HCl}$ in bestimmter Menge zugefügt und das ausgeschiedene Jod mit Schwefelkohlenstoff ausgeschüttelt wurde. Die einzelnen Ausschüttelungen wurden von anlaftendem Jodat durch Waschen befreit, vereinigt und mit $1 / 10$ N.- $\mathrm{Na}_{2} \mathrm{~S}_{2} \mathrm{O}_{3}$ titrirt; ebenso wurde der vorhandene Jodatrest bestimmat durch Versetzen mit überschüssiger Kaliumjodidlösung und Schwefelsäure und Ermittelung des frei gewordenen Jods.

Je $20 \mathrm{ccm}$ einer Lösung, enthaltend $0,32095 \mathrm{~g}$ K.J und ihrem Jodgehalte nach $19,93 \mathrm{~cm}$ einer $1 / 10 \mathrm{~N} \cdot-\mathrm{Na} \mathrm{S}_{2} \mathrm{~S}_{2} \mathrm{O}_{3}$ entsprechend, und $25 \mathrm{ccm}$ einer Lösung, enthaltend $0,10136 \mathrm{~g}$ $\mathrm{KJO}_{3}$ und ihrem Wirkungswerthe nach $29,29 \mathrm{ccm}^{1 / 10} \mathrm{~N} .-\mathrm{Na}_{2} \mathrm{~S}_{2} \mathrm{O}_{3}$ entsprechend, wurdeu zu einem Versuche verwendet. Die Jodmenge, welche bei Zusatz der theoretischen Säuremenge frei werden soll, entspricht dem im zugesetzten Kaliumjodid enthaltenen Jod, vermehrt um $1 / 5$ dieser Menge, entsprechend dem bei der Reduction des Jodats freiwerdenden Jod, rerbraucht also theoretisch $19,93+3,99=23,92 \mathrm{ccm} 1 / 10 \mathrm{~N} .-\mathrm{Na}_{2} \mathrm{~S}_{2} \mathrm{O}_{3}$ $\left(1 \operatorname{ccm}^{2} / 10 \mathrm{~N} .-\mathrm{Na}_{2} \mathrm{~S}_{2} \mathrm{O}_{3}=0,012307 \mathrm{~g} . \mathrm{J}\right)$. Die theoretische Menge an zuzusetzender $1 / 10 \mathrm{~N}$.$\mathrm{HCl}$ beträgt $20,35 \mathrm{ccm}(1 \mathrm{ccm} \mathrm{HCl}=$ $0,00416 \mathrm{~g} \mathrm{HCl})^{*}$ ).

\begin{tabular}{c|c|c|c}
\hline $\begin{array}{c}\mathrm{ccm} \\
1 / 10 \mathrm{~N} .-\end{array}$ & $\begin{array}{c}\mathrm{ccm}{ }_{\mathrm{HCl}} / 10 \mathrm{~N} .- \\
\mathrm{Na}_{2} \mathrm{~S}_{2} \mathrm{O}_{3} \text { entspr. } \\
\text { d. freigew. J }\end{array}$ & $\begin{array}{c}\mathrm{cm} \mathrm{Cm}_{10} \mathrm{~N} .- \\
\mathrm{Na}_{2} \mathrm{~S}_{2} \mathrm{O}_{3} \text { entspr. } \\
\text { dem Jodatreste }\end{array}$ & $\begin{array}{c}\text { Summe von } \\
\text { verbr. ecm } \\
1 / 10 \mathrm{~N} . \mathrm{Na}_{2} \mathrm{~S}_{2} \mathrm{O}_{3}\end{array}$ \\
\hline 19,60 & 23,15 & 6,10 & 29,25 \\
20,20 & 23,60 & 5,65 & 29,25 \\
20,35 & 23,75 & 5,60 & 29,35 \\
21,40 & 23,85 & 5,45 & 29,30
\end{tabular}

Um durch die zugefügte Säure kein HaJogen in die Lösung zu bekommen, wurde statt der Salzsäure nun 1/, N N.-Schwefelsäure verwendet. $\mathrm{Zu}$ den folgenden Versuchen kamen frisch bereitete ungefähr 1/10 N.-Lösungen in Anwendung; gleichzeitig wurde versucht, statt des Schwefelkohlenstoffs als Ausschüttelungsflüssigkeit Toluol zu verwenden, da das Arbeiten mit ersterem wegen der grossen Flüchtigkeit, des üblen Geruches etc. mit Unannehmlichkeiten verbunden ist.

*) Die verwendeten Lösungen sind, wie aus der Angabe der Titer ersichtlich ist, nur annähernd $1 / 10$ normal. 
$25 \mathrm{ccm}$ ciner beil äufig $1 / 10$ N.-Kaliumjodidlösung wurden mit $50 \mathrm{ccm}$ einer annähernd $1 / 10 \mathrm{~N}$-haliumjodatlösung und mit $1 / 10 \mathrm{~N}$. $-\mathrm{H}_{2} \mathrm{SO}_{4}$ in geringem Überschusse versetzt. Bei zwei Versuchen betrug der Verbrauch an $1 /{ }_{10} \mathrm{~N}$.$\mathrm{Na}_{2} \mathrm{~S}_{3} \mathrm{O}_{3}$ für das freigewordene Jod 29,85) und $29,75 \mathrm{ccm}$ (theoretisch $29,78 \mathrm{~cm}),(1 \mathrm{ccm}$ $1 / 10 \mathrm{~N}^{2}-\mathrm{Na}_{3} \mathrm{~S}_{2} \mathrm{O}_{3}=0,0128696 \mathrm{~g} \mathrm{~J}, 1 \mathrm{ccm}{ }^{1 / 10} \mathrm{~N}$.$\mathrm{KJO}_{3}=0,035653 \mathrm{~g} \mathrm{KJO}_{3}, 1 \mathrm{ccm} 1 / 10 \mathrm{~N}$.-K.J $=0,01673 \mathrm{~g} \quad \mathrm{JK}$ ). Die Anwendung des Toluols als Ausschüttelungsmittel erwies sich in vielfacher Hinsicht als vortheilhaft, so dass wir auch bei den folgenden Versuchen dasselbe zur Anwendung brachten.

Von Interesse ,war es nun, das Verhalten von Jodid gegen Jodat bei Gegenwart einer schwachen Säure, wie Essigsäure, zu untersuchen, bei deren Anwendung die an und für sich geringe Concentration der Wasserstoffionen noch dureh Zusatz yon verschieden grossen Mengen ihres Neutralsalzes, Natriumacetat, entsprechend herabgedrückt werden $\left.\operatorname{kann}^{8}\right)$.

Es wurden zuerst je $10 \mathrm{ccm}$ obiger KJLösung mit $20 \mathrm{ccm}$ der $\mathrm{KJO}_{3}-\mathrm{Lösung}$ versetzt und bei einem Versuche $20 \mathrm{ccm}$ Eisessig und $20 \mathrm{cem}$ Wasser, bei einem zweiten $20 \mathrm{ccm}$ Essigsäure (ca. 20 proc.) und $20 \mathrm{ccm}$ Wasser zugefügt, das ausgeschiedene $J$ mit Toluol ausgeschüttelt und mit $1 / 10 \mathrm{~N}$. $-\mathrm{Na}_{2} \mathrm{~S}_{2} \mathrm{O}_{3}$ titrirt. Der Verbrauch an letzterem betrug 11,95 bez. $11,9 \mathrm{ccm}$ (theor. 11,91 ccm). Der in der wässerigen Lösung verbliebene Jodatrest entsprach in beiden Fällen $7,75 \mathrm{ccm}$ $1 / 10 \mathrm{~N} .-\mathrm{Na}_{2} \mathrm{~S}_{2} \mathrm{O}_{3}$ (theor. $7,74 \mathrm{ccm}$ ). Wurden bei Anwendung von $25 \mathrm{ccm} J \mathrm{~K}$ und $25 \mathrm{cem}$ ${\mathrm{K} . J \mathrm{O}_{3}}_{3}$ neben $40 \mathrm{cem}$ Essigsäure $10 \mathrm{~cm} \mathrm{Na-}$ triumacetat (25 Proc.) zugefügt, so war der Reactionsverlauf ein unvollständiger, indem statt $29,78 \mathrm{~cm}{ }^{1} /{ }_{10} \mathrm{~N} .-\mathrm{Na}_{3} \mathrm{~S}_{2} \mathrm{O}_{3}$ bei 3 Versuchen nur $27,9,27,85,27,95 \mathrm{ccm} 1 / 10 \mathrm{~N} .-\mathrm{Na}_{2} \mathrm{~S}_{2} \mathrm{O}_{3}$ für das ausgeschütteltc Jod, hingegen 19,8, $19,6,19,5 \mathrm{ccm}^{1 / 10} \mathrm{~N}$.- $\mathrm{Na}_{3} \mathrm{~S}_{2} \mathrm{O}_{3}$ für den Jodatrest (statt $19,32 \mathrm{ccm}$ ) verbraucht wurden. Nicht aufgeklärt ist hierbei, dass der Gesammtverbrauch an $1 / 10 \mathrm{~N}$. $-\mathrm{Na}_{2} \mathrm{~S}_{2} \mathrm{O}_{3}$ statt $49,10 \mathrm{ccm}$, entsprechend dem angewendeten Jodate, hier nur $47,7,47,35,47,45 \mathrm{~cm}$ beträgt. Diese Versuche lassen crsehen, dass bei Gegenwart von $10 \mathrm{ccm}$ Natriumacetat (25 Proc.) $40 \mathrm{ccm}$ Essigsäure nicht genïgen, um die theoretische Menge an Jod, cntsprechend einer vollständigen Oxydation dess vorhandenen Jodids, zu erhalten. Wird die das über-

${ }^{8}$ ) Gunner Jörgensen (Chem. Ztg. Repert. 1897, 247) hat die Reactionsschnelligkeit bei der Einwirkung verschiedener organischer Säuren auf Jodat-Jodid mittels des jodometrischen Säurebetrags gemessen und auch in einigen Fällen den verzögernden Einfluss der Neutralsalze constatirt. schüssige Jodat enthaltende wässerige Lösung vor dem Zusatze vom Jodkalium mit verdünnter Schwefelsäure iw Überschusse versetzt, so tritt Jodausscheidung auf, was auf noch vorhandenes, unzersetzt gebliebenes Jodid zurïckzuführen ist. Bei einigen folgenden Versuchen verminderten wir die Menge des Natriumacetates und constatirten, dass dann der Reactionsverlauf mit abnehmendem Gehalte der Lösung an Natriumacetat sich dem quantitativen nähert, also eine fast vollständige Ausscheidung des Jods des vorhandenen Jodkaliums stattfindet. Doch war auch bei Anwesenheit von relativ geringen Mengen von Natriumacetat, nach dem Gesammtverbrauche an $1 / 10$ N.- $\mathrm{Na}_{2} \mathrm{~S}_{2} \mathrm{O}_{3}$ zu schliessen, auch hier ein nicht erklärter VerIust an Jodat in der von Jod durch Ausschütteln befreiten, wässerigen Lösung zu constatiren.

Da nach den oben angegebenen Versuchen die durch Essigsäure und Natriumacetat in bestimmten Mengenverhältnissen bei Anwendung von überschüssigem Jodat freigewordene Jodmenge der theoretisch erwarteten nicht in allen Fällen vollständig entsprach, wurde nun der Reactionsverlauf in der Wärme untersucht, in der Weise, dass das in Freiheit gesetzte Jod durch Erhitzen auf dem Drahtnetze verjagt und der zurückbleibende unzersetzte Jodatrest, wie gewöhnlich, ermittelt wurde. Bei Anwendung ron $50 \mathrm{ccm}$ $\mathrm{KJO}_{3}, 25 \mathrm{ccm} \mathrm{KJ}, 40 \mathrm{ccm}$ Essigsäure und $20 \mathrm{ccm}$ Natriumacetat wurden für den Jodatrest $13,05 \mathrm{ccm} 1 / 10 \mathrm{~N} .-\mathrm{Na}_{2} \mathrm{~S}_{2} \mathrm{O}_{3}$ verbraucht, während theoretisch demselben $19,32 \mathrm{ccm}$ entsprechen sollten. Diese unerwartete Abweichung ron dem theoretischen Werthe veranlasste uns, auch mit Hinsicht auf die bei den früheren Ausschüttelungsversuchen beobachteten, unaufgeklärten Jodatverluste, nun die Versuche in der Wärme nur mit Essigsäurc, also ohne Natriumacetatzusatz, vorzunehmen.

$50 \mathrm{ccm}$ der $\mathrm{KJO}_{3}$-Lösung und $25 \mathrm{ecm}$ der KJ-Lösung wurden mit $40 \mathrm{ccm}$ Essigsäure versetzt, bis zur völligen Austreibung des freien Jods gekocht und der Jodatrest bestimmt. Für denselben wurden bei zwei Versuchen 14,35 und $12,65 \mathrm{ccm}{ }^{1} / 10 \mathrm{~N} .-\mathrm{Na}_{2} \mathrm{~S}_{2} \mathrm{O}_{3}$ (theoretisch 19,32 $\mathrm{ccm}$ ) verbraucht; es ergab sich also wieder eine ähnliche Abweichung von dem theoretischen Werthe wie fräher. Um zu sehen, ob, was zwar nicht anzunehmen war, vielleicht eine Einwirkung der Essigsäure auf das Kaliumjodat in der Wärme stattfinde, wurden $50 \mathrm{ccm} \mathrm{KJO}_{3}$ mit $40 \mathrm{ccm}$ Essigsäure längere Zeit erhitzt, hierbei aber keinerlei Verlust an $\mathrm{KJO}_{3}$ beobachtet, indem nach dem Erkalten und bei Zusatz von 
überschüssigem Jodkalium und Säure $49,1 \mathrm{ccm}$ $1 / 10 \mathrm{~N} .-\mathrm{Na}_{2} \mathrm{~S}_{2} \mathrm{O}_{3}$, die theoretische Menge, verbraucht wurden. Ein zweiter Versuch mit $20 \mathrm{cem} \mathrm{KJO}_{3}, 20 \mathrm{cem}$ Eisessig und $20 \mathrm{~cm}$ Wasser ausgeführt, ergab wieder die richtige Menge von $19,65 \mathrm{ccm} \quad 1 / 10 \mathrm{~N} .-\mathrm{Na}_{2} \mathrm{~S}_{2} \mathrm{O}_{3}$. Wurden unter den gleichen Umständen neben $20 \mathrm{ccm} \quad \mathrm{KJO}_{3} 10 \mathrm{ccm}$ JK angewendet, so wurde nur $1 \mathrm{ccm} 1 / 10 \mathrm{~N} .-\mathrm{Na}_{2} \mathrm{~S}_{2} \mathrm{O}_{3}$ für das restliche Jodat verbraucht, während nach der Menge des zugesetzten Jodkaliums bei normalem Reactionsverlaufe $7,75 \mathrm{~cm}$ 1/10 N.$\mathrm{Na}_{2} \mathrm{~S}_{2} \mathrm{O}_{3}$ verbraucht werden sollten.

Um den Einfluss des vorhandenen Jodkaliums bei dem anormalen Reactionsverlaufe kennen zu lernen, wurde die Menge an zugesetztem $\mathrm{KJ}$ verringert. Bei Anwendung von $20 \mathrm{ccm} \mathrm{KJO}_{3}, 1 \mathrm{ccm} \mathrm{KJ}, 20 \mathrm{ccm}$ Eisessig, $20 \mathrm{ecm} \mathrm{H}_{2} \mathrm{O}$, entsprechend dem Jodatreste $17,25 \mathrm{~cm} 1 / 10 \mathrm{~N} .-\mathrm{Na}_{2} \mathrm{~S}_{2} \mathrm{O}_{3}$ (statt 18,46 ccm); $20 \mathrm{ccm} \mathrm{KJO}, 5 \mathrm{~cm} \mathrm{K.J,} 20 \mathrm{ccm}$ Eisessig, $20 \mathrm{ccm} \mathrm{H}_{2} \mathrm{O}$, entsprechend dem Jodatreste $7,40 \mathrm{ccm}{ }_{1 / 10}^{1 /} \mathrm{N} .-\mathrm{Na}_{2} \mathrm{~S}_{2} \mathrm{O}_{3}$ (statt $13,70 \mathrm{ccm}$ ). 1 ecm $\mathrm{KJ}$ sollte $\mathrm{KJO}_{3}$, entsprechend $1,19 \mathrm{crm}$ $1 / 10 \mathrm{~N}$ - $\mathrm{Na}_{2} \mathrm{~S}_{2} \mathrm{O}_{3}$, verbrauchen, es ist aber $\mathrm{KJO}_{3}$, entsprechend $2,40 \mathrm{ccm} 1 / 10 \mathrm{~N} .-\mathrm{Na}_{2} \mathrm{~S}_{2} \mathrm{O}_{3}$, verschwunden; $5 \mathrm{ccm}$ KJ sollte $\mathrm{KJO}_{3}$, entsprechend $5,95 \mathrm{ccm} 1 / 10 \mathrm{~N} .-\mathrm{Na}_{2} \mathrm{~S}_{3} \mathrm{O}_{3}$, verbrauchen, es ist aber $\mathrm{KJO}_{3}$, entsprechend $12,25 \mathrm{ccm} 1 / 10 \quad$ N.- $-\mathrm{Na}_{2} \mathrm{~S}_{2} \mathrm{O}_{3}$, verschwunden. Wie aus diesen Zahlen zu ersehen ist, wurde bei diesen Versuchen beiläufig die doppelte Menge an Jodat reducirt, als entsprechend dem vorhandenen Jodion erforderlich wäre. Von dem vollständigen Oxydationsverlaufe, also von der Abwesenheit von Jodkalium in der erkalteten Lösung, überzengten wir uns bei einigen Versuchen durch Zusatz ron überschüssiger Schwefelsäure, bevor wir .Jodkalium zusetzten; es trat hierbei kein Freiwerden von Jod ein.

Um festzustellen, ob entsprechend dem Mehrverbrauche an Jodat die freiwerdende Jodmenge grösser ist, als sie entsprechend dem zugesetzten Jodkalium sein sollte, wurde das beim Erhitzen entweichende Jod in einer mit Jodkalium beschickten Vorlage aufgefangen und bestimmt. Bei Anwendung von $20 \mathrm{~cm} \mathrm{KJO}_{3}, 10 \mathrm{~cm} \mathrm{KJ}, 20 \mathrm{~cm}$ Fisessig und $20 \mathrm{ccm} \mathrm{H}_{2} \mathrm{O}$ wurde das freigewordene Jod mit $12,15 \mathrm{ccm} 1 / 10 \mathrm{~N} .-\mathrm{Na}_{2} \mathrm{~S}_{2} \mathrm{O}_{3}$ titrirt. Wurden bei gleicher Jodkaliummenge $50 \mathrm{ccm} \mathrm{KJO}_{3}$ angewendet, so betrug der Verbrauch an ${ }^{1} / 10$ N. $-\mathrm{Na}_{2} \mathrm{~S}_{2} \mathrm{O}_{3} 12,0 \mathrm{ec} \cdot \mathrm{m}$. Da theoretisch $11,91 \mathrm{ccm}^{1} / 10 \mathrm{~N} .-\mathrm{Na}_{2} \mathrm{~s}_{3} \mathrm{O}_{3}$ verbraucht werden sollten, so war erwiesen, dass nur die dem Jodkalium entsprechende Jodmenge frei wird, unabhängig von dem zugesetzten Überschusse an Kaliumjodat.

Aus den früher angeführten Versuchen ist ersichtlich, dass der Mehrverbrauch an Kaliumjodat abhängig von der Menge des angewendeten Jodkaliums ist. Da aber das vorhandene Jodkalium, wie ebenfalls aus den schon gemachten Angaben zu ersehen ist, bei Gegenwart von überschüssigem Jodate und Essigsäure sofort vollständig oder fast vollständig Oxydation erfährt, also Jodionen in der Lösung in irgendwie in Betracht kommender Menge schon vor dem Erhitzen nicht mehr vorhanden sind, so lag die Ansicht nahe, dass die Menge des entstehenden elementaren Jods die Reaction beeinflusst. Einige Versuche ergaben die Richtigkeit dieser Schlussfolgerung, indem bei Anwendung von $20 \mathrm{ccm} \mathrm{KJO}_{3}, 20 \mathrm{ccm}$ Eisessig und $20 \mathrm{ccm}$ Wasser ohne Zugabe von Kaliumjodid, dagegen bei Zusatz von steigenden Mengen an festem Jod der Verbrauch an Thiosulfat zur Bestimmung des restlichen Jodats abnahm. Der anormale Reactionsverlauf scheint also auf eine Einwirkung des Jods bei Gegenwart von Jodat auf die Essigsäure zurückzuführen zu sein, was durch weitere Untersuchungen, die sich auch auf eine Reihe von anderen organischen Säuren erstrecken werden, festgestellt werden soll. Dieselben werden sich vorerst in der Richtung bewegen, die Jodat, Jodid und Essigsäure enthaltende Lösung unter Anwendung eines Kühlers zu erhitzen, so dass das Jod nicht wie bei den früheren Versuchen aus der Lösung während des Erhitzens entfernt wird. Es dürfte dann möglich sein, neben dem freien $J_{o d}$ und dem überschüssigen Jodat auch direct die Jodmenge zu bestimmen, welche wahrscheinlich in einer organischen Verbindung vorhanden ist, event. auch letztere und damit den Chemismus der stattfindenden Reaction näher zu charakteri$\operatorname{siren}^{9}$ ).

Um den Einfluss der Wasserstoffionenconcentration bei der Oxydation der Halogenide durch Jodat noch näher zu studiren, wurde nun noch die Wirkung der schwächsten Säuren in Betracht gezogen. Wird in eine Jodat und Jodid enthaltende Lösung Kohlensäure eingeleitet, so wird bekanntlich durch beigefügte Stärkelösung in der Flüssigkeit schon nach sehr kurzer Zeit das Auftreten von freiem Jod angezeigt. Die Menge des freigewordenen Jods ist aber auch nach längerer Einwirkung nur eine relativ geringe, wahr-

$\left.{ }^{9}\right)$ Nach der Methode von Kékulé erhält man Jodessigsäure, wenn man ein Gemenge von Essigsäureanhydrid, Jod und Jodsäure zum Sieden bringt. (Vergl. Jahresber. über d. Fortschritte d. Chemie 1868, 505.) Möglicherweise findet hier secundär, also nach dem Freiwerden des Jods und beim darauffolgenden Erhitzen, der gleiche Vorgang statt. 
scheinlich deshalb, weil in Folge der geringen Löslichkeit der Kohlensäure unter Atmosphärendruck die Concentration der Wasserstoffionen dementsprechend selır gering ist. Bei Anwendung von $25 \mathrm{ccm}{ }^{1 / 10} \mathrm{~N}$.- $\mathrm{KJO}_{3}$ und $20 \mathrm{ccm}{ }^{1} / 10$ N.- JK entspricht nach 10 Minuten langem Finleiten eines mässig raschen Kohlensäurestromes, der durch eine Lösung von kohlensaurem Natron von etwa mitgerissenen Salzsäurespuren befreit worden war, die Menge des freigewordenen Jods $1,4 \mathrm{ccm} 1 / 10$ N.-Thiosulfat $^{10}$ ). Wie $z u$ erwarten ist, war unter gleichen Umständen, also bei Einwirkung von Kohlensäure auf ein Gemisch von Jodat und Bromid bez. Jodat und Chlorid, das Auftreten von freiem Halogen nicht $z u$ bemerken.

Wird einer mit Stärke versetzten Lösung von Jodat und Jodid eine wässerige, mässig verdünnte Lösung von $B$ ors äure zugesetzt, so wird keine Spur Jod frei. Die Wasserstoffionenconcentration der Borsäure in wässeriger Lösung von der angewendeten Concentration erreicht demnach nicht jene.Grösse, um auch nur spurenweise den Übergang des Jodions in elementares Jod zu ermöglichen, was auch mit der Thatsache in Übereinstimmung steht, nach welcher die wässerige Lösung der Borsäure nur schwach sauer reagirt und die Elektricität nur wenig besser als reines Wasser leitet $\left.{ }^{11}\right)$. Wird aber der mit Borsäure versetzten Lösung von Jodat und Jodid etwas Glycerin zugefügt, so tritt, bei relativ grösserer Menge an letzterem sofort, bei sehr geringen Mengen nach einiger Zeit, Ausscheidung von Jod ein. Die Reaction beruht auf der bekrnnten Eigenschaft der Borsäure, mit mehrfach hydroxylirten organischen Verbindungen complexe Säuren zu bilden, die eine bedeutend stärker saure Reaction zeigen als die Borsäure bez. die zugesetzte organische Substanz selbst. Wird der Jodat und Jodid enthaltenden Lö-

10) Die auf die Ausscheidung von Jod zurückzuführende Gelbfärbung bei längerer Zeit der atmosphärischen Luft ausgesetzten Jodkaliumlösungen ist, wie schon bekannt, auch auf den Einfluss der Kohlensäure und der nachfolgenden Einwirkung des Luftsauerstoffes zurückzuführen.

11) Nach J. Walker u. W. Cormack (Proc. Chem. Soc. 15, 208, Ref. Zeitschr. f. anorg. Ch. 1900, 23, 229) beträgt die Ionenspaltung in $1 / 10$ N.-Lösungen von Kohlensäure 0,174 Proc., Borsäure 0,013 Proc., Phenol 0,0037 Proc. Es ist also nach diesen Angaben, die in $1 / 10$ N.-Lösung der Borsäure vorhandene Wasserstoffionenconcentration eine üusserst geringe. Wird aber eine concentrirtere Lösung von Borsiure zu einer Jodat und Jodid enthaltenden Lösung hinzugefügt, so tritt schon nach kurzer Zeit eine geringe Jodausscheidung auf. Uber das Verhalten der Borsäure zu Jodat-Jodid in wässeriger Lösung für sich und bei Gegenwart von Glycerin bez. Mannit liegt auch 1. A. eine Mittheilung von L. C. Jones (Zeitschr. f. anorg. Ch. 20, 213, 21, 169) vor. sung Glycerin allein zugesetzt, so tritt natürlich keine Jodausscheidung auf. Setzt man nebst Borsäure statt Glycerin Dextrose der Lösung zu, so tritt ebenfalls Jodausscheidung, wenn auch im relativ geringeren Maasse als bei Zusatz von Glycerin auf ${ }^{12}$ ). Das Freiwerden von Jod aus einem Gemisch von Jodat und Jodid durch Tusatz von Borsäure und Glycerin könnte benützt werden, um in gewissen Fällen Glycerin qualitativ nachzuweisen; ob es auch möglich sein wird die Reaction unter Einhaltung bestimmter Verbältnisse, wie Concentration des Jodats, Jodids und der Borsüure, und der Einwirkungsdauer, als Grundlage zu einer quantitaven Bestimmung des Glycerins auf jodometrischem Wege zu verwenden können erst weitere Versuche zeigen. Natürlicherweise müsste dann die Anwesenheit aller Jod in Freiheit setzenden Substanzen von vornherein ausgeschlossen sein.

Interessant ist auch das Verhalten der Carbolsäure gegenüber Jodat-Jodid. Wird eine wässerige Lösung von Phenol zu einer Lösung von Jodat und Jodid, die mit Stärkelösung versetzt ist, hinzugefügt, so tritt gar keine Jodausscheidung auf. Trotz der Eigenschaft des Phenols, sich ähnlich einer Säure mit Alkalien zu salzähnlichen Verbindungen, den Phenolaten, zu vereinigen, enthält seine wässerige Lösung keine oder nur sehr geringe Mengen von Wasserstoffionen. In Übereinstimmung hiermit steht auch der Umstand, dass die Phenolate in wässeriger Lösung sehr stark hydrolytisch dissociirt sind. Der saure Charakter der Phenole wird, wie bekannt, bedeutend erhöht, wenn in den Benzolkern Nitrogruppen eingeführt werden. Dementsprechend tritt bei Zusatz einer wässerigen Lösung von Pikrinsäure (Trinitrophenol) zu einer Jodat und Jodid enthaltenden Lösung sofort eine bei Gegenwert von Stärkelösung sichtbare Jodausscheidung auf; dieselbe tritt schon beim Zufügen von geringen Mengen Pikrinsäure auf, ist aber auch bei grösserem Gehalt der Lösung an letzterer nur eine relativ geringe. Pikrinsäure, nur mit Jodkalium und Stärkelösung versetzt, giebt natürlich keine Jodausscheidung.

Wir haben schon oben angegeben, dass, zum Unterschiede von Jodid, Bromid durch Kaliumjodat selbst bei Zusatz einer grösseren Menge an $1 / 10$ N.- bez, $1 / 3$ N.-Salzsäure nicht oxydirt wird, also eine bestimmte Menge von Wasserstoffionen nothwendig ist, um entsprechend der geringeren Ionisirungstendenz

12) Wird eine Lösung von Bromid und Jodat mit Borsäure und Glycerin versetzt, so erfolgt auch bei grösseren Meugen an letzterem keine Ausscheidung von freiem Halogen. 
des Broms die Überführung des Ions in das elementare Brom herbeizuführen. Mit Hinsicht auf die mit Essigsäure bei Jodid erhaltenen Resultate untersuchten wir aun anch das diesbezügliche Verhalten des Jodats gegenüber Bromid. Eine 10 proc. Bromidlösung, mit überschüssigem Jodat, $10 \mathrm{ccm}$ Natriumacetat und $80 \mathrm{ccm}$ Essigsäure versetzt, zeigte auch nach längerer Jinwirkungsdauer kein Freiwerden von Brom und Jod. Dasselbe fand auch bei Anwendung von $50 \mathrm{ccm}$ lissigsäure ohne Zusatz von Natriumacetat statt. Wurde aber die essigsaure Lösung zum Kochen crhitzt, so trat Oxydation des Bromids, wenn auch in sehr geringem Maasse, rin.

Es wurden nun auch einige orientirende Versuchc durchgeführt, bei welcheu die Oxydation des Jodids durch Jodat und Essigsäure bei Gegenwart von Bromid quantitativ verfolgt wurde.

$10 \mathrm{~cm} 1 / 30 \mathrm{~N} . \mathrm{KJ}$ und $5 \mathrm{ccm} / / 10 \mathrm{~N}$ $\mathrm{KBr}$, mit $20 \mathrm{~cm} 1 / 10 \mathrm{~N} .-\mathrm{KJO}_{3}, 20 \mathrm{ccm}$ Essigsäure und $20 \mathrm{ccm}$ Wasser versetzt und mit Toluol ausgeschüttelt, ergaben eine Jodausscheidung, entsprechend $11,85 \mathrm{ecm} 1 / 10$ N.$\mathrm{Na}_{2} \mathrm{~S}_{2} \mathrm{O}_{3}$, während dem vorhandenen . Todide nach 11,91 cem verbraucht werden sollten. Der Versuch, unter sonst gleichen Tmständen mit $15 \mathrm{ccm} 3 / 10$ N.-Bromid durchgefïhrt, ergab einen Verbrauch von $11,80 \mathrm{~cm} 1 / 10$ N.$\mathrm{Na}_{2} \mathrm{~S}_{2} \mathrm{O}_{3}$. Der Jodatrest entsprach bei den zwei Versuchen 7,7 bez. $7,8 \mathrm{ccm} 1 / 10 \mathrm{~N} .-\mathrm{Nat}_{2} \mathrm{~S}_{2} \mathrm{O}_{3}$ (theoret. $7,74 \mathrm{ccm}$ ).

Weiter wurden $25 \mathrm{ccm} 1 / 10$ N.-KJ, $25 \mathrm{ccm}$ $1 / 10 \mathrm{~N} .-\mathrm{KBr}$ mit $50 \mathrm{ecm} \mathrm{KJO}_{3}$ eine Stunde lang im Scheidetrichter einmal mit 20, ein zweites Mal mit $60 \mathrm{ccm}$ Eisessig stehen gelassen, dann mit Toluol ausgeschättelt und das in Lösung gegangene Jod sowie cler Jodatrest bestimmt. In beiden Fällen ergatb sich für das erstere $29,75 \mathrm{ccm} 1 / 10 \quad \mathrm{~N} .-\mathrm{Na}_{3} \mathrm{~S}_{2} \mathrm{O}_{3}$ (theoret. 29,78), für den Jodatrest aber hei Anwendung von $20 \mathrm{ccm}$ Eisessig $19,25 \mathrm{ccm}$, bei $60 \mathrm{~cm}$ liisessig $17,1 \mathrm{~cm} \mathrm{Na} \mathrm{S}_{2} \mathrm{O}_{3}$ (the0ret. $19,32 \mathrm{ccm})$. Bei grösserem Essigsäureüberschuss und einer Linwirkungsdauer von einer Stunde fand also augenscheinlich auch eine geringe Reduction des Jodates durch das vorhandene Bromid statt ${ }^{13}$ ).

Es wurde nun noch ein Versuch durchgeführt, bei welchem an Stelle der Essigsüure $1 / 10$ N.-Salzsäure in Verwendung kam. Bei Anwendung von $25 \mathrm{cem} \mathrm{KJ,} 25 \mathrm{cem} \mathrm{K} \mathrm{Br}$, $50 \mathrm{ccm} \mathrm{KJO}_{3}$ und $25,5 \mathrm{ccm} 1 / 10 \mathrm{~N} .-\mathrm{HCl}$ wurden $29,7 \mathrm{ccm}$ (theoret. $29,78 \mathrm{ccm}$ ) für das

13) Das hierbei freiwerdende Brom dürfte von dem zugesetzten Toluol (oder auch der Essigsäure) substitutiv aufgenommen werden, während die geringe Menge des gleichzeitig freiwerdenden Jods zahlenmässig wenig in Betracht kommt. ausgeschiedene Jod und $19,45 \mathrm{ccm}$ (theoret. $19,32 \mathrm{ccm}){ }_{1}^{1} / 10 \mathrm{~N} .-\mathrm{Na}_{3} \mathrm{~S}_{2} \mathrm{O}_{3}$ für den Jodatrest verbraucht.

Die angeführten Versuche gestatten den Schluss, dass es möglich sein wird, ein einfaches und exactes Verfahren zur Bestimmung von Jodiden neben Bromiden bez. Jodiden neben Chloriden auf das verschiedene Verhalten der Halogenide gegen Jodat bei bestimmter Concentration der Wasserstoffionen zu basiren. Die Menge des vorhandenen Jods könnte suwohl direct durch Bestimmung des ausgeschüttelten Jods oder aber auch indirect durch Bestimmung des Jodatrestes ermittelt werden $\left.{ }^{3 t}\right)$. Nach einigen Vorversuchen dürfte es auch möglich sein bei Anwendung bestimmter Säuren den Jodlatrest nach Entfernung des Jods durch Erhitzen zu bestimmen und dürfte dies, selbst im Falle hierbei ein Angriff von Bromid bez. Chlorid stattfindet, zu einem leicht durchführbaren Verfahren zur Bestimmung von Jodiden als solchen führen ${ }^{15}$ ).

Die Reaction zwischen Jodsäure und Bromwasserstoff geht, wie sehon erwähnt, nach Angabe von Bugarszky (loc. cit.) bei gewöhnlicher Temperatur suhr langsam vor sich und erhält man nach ihm selbst aus roncentrirten Lösungen das ausgeschiedene Brom und Jod nicht quantitativ, wenn min die letzteren mit Chloroform oder Schwefelkohlenstoff ausschüttelt. Um den bei gewöhnlicher Temperatur stattfindenden Reactionsverlauf bei verschiedenen Concentrationen und Anwendung wechselnder Säuremengen ü̈her zu studiren, ist die Bestimmung der ausgeschiedenen Halogene durch Ausschüttelung nicht ganz einwandfrei, da selbst bei der Annahme, dass das Ausschüttelungsmittel durch Brom gar keinen Angriff erleidet, Bromverluste durch Verdampfen kaum ver-

14) Wir wollen hier erwähnen, dass F.W.K üster (Ref. Z. f. plyssik. Chemie 1898, 377 ) durch stufenweise Auderung der Wasserstoffionenconcentration bei Anwendung von Kaliumpermanganat aus einer Lösung, die J-, Br- und Cl-Ionen neben einander enthält, jedes der Halogene für sich abscheidet. Siehe diesbezüglich auch $P$. Jannasch und K. Aschoff (Z. f. anorg. Chemie 1892, 1, 144). Speciell den Einfluss von essigsauren Salzen (Natrium- oder Bleiacetat) bei der Trennung ron $\mathrm{Br}$ und $\mathrm{Cl}$ durch Bleisuperoxyd und Essigsäure hat schon G. Vortmann (Z. f. analyt. Chemie 1886, 173) erkannt, indem er angiebt, dass bei Anwesenheit von Acetat Bromide durch 5-proc. Essigsäure und Bleisuperoxyd gar keine Zersetzung erfahren und erst beim Kochen mit der 25-proc. Essigsäure Spuren von $\mathrm{Cl}$ frei werden. Doch giebt erst Kü ster die diesbezügliche theoretische Erklärung.

15) Bezüglich der Anwendung der Jodsäure bez. des Jodats zur Bestimmung von Jodiden, siehe auch E. Riegler (Zeitschr. f. analyt. Chemie 1896, 305), Gooch n. Walker (Zeitschr. f. anorg. Chemie $1897, \mathbf{1 4}, 422)$. 
mieden werden können. Ob man nun bei der Reduction der Jodsäure durch Bromwasserstoff das Oxydations- oder das Reductionsmittel im Überschusse anwendet, kann in keinem Falle, wie leicht einzusehen ist, das freigewordene Brom (und Jod) auf dem üblichen Wege, nämlich durch Umsetzung mit Jodkaliumlösung und Ermittelung des abgeschiedenen Jods mittels Thiosulfat bestimmt werden, da ja sonst die unzersetzt gebliebene Jodsäure vollständig Reduction erfahren würde. Um die Menge der unter den jeweiligen Umständen ausgeschiedenen freien Halogene zu ermitteln, ohne eine weitere Reduction des überschüssigen bez. unzersetzten Jodats herbeizuführen, käme vielleicht nach einigen von uns durchgeführten Vorversuchen der folgende Weg in Betracht.

Die die freien Halogene sowie das überschüssige Jodat enthaltende saure Lösung könnte nach entsprechender Verdünnung mit Kalilauge oder kohlensaurem Alkali bis fast zur Neutralisation versetzt werden ${ }^{16}$ ), dann eine mit überschüssigem Bicarbonat versetzte Lösung von $1 / 10$ N.-Natriumarsenit im Überschusse zugesetzt und dieser mit 1/10 N.-Jodlösung ermittelt werden. Festgestellt müsste lierbei vorerst werden, ob eine quantitative Unsetzung zwischen den freien Halogenen bez. dem primär gebildeten Hypobromit und Irypojodit und dem Arsenit stattfindet, also bei den eingehaltenen Umständen keine Halogenatbildung eintritt. Selbstverständlich müsste die Neutralisation sowie die Zugabe von Natriumarsenit bei völliger Vermeidung von Bromverlusten stattfinden, was bei Anwendung des von einem von uns bei einer anderen Gelegenheit angegebenen Apparates ${ }^{17}$ ) ermöglicht ist ${ }^{18}$ ).

16) Als Indicator könnte vielleicht ein geringer Zusatz eines Salzes, wie Zink-oder Calciumsulfat, dienen, welche mit überschüssigem Carbonat eine Fällung ergeben.

17) H. Ditz und H. Knöpfelmacher (Z. f. angew. Chemie $1899,1195,1217$ ) and Il. Ditz (Chemiker-Ztg. 1901, 727). Der dort zur Untersuchung von Chlorat-Hypochloritgemischen angegebene Apparat besteht aus einer Schüttelflasche mit einem eingeschliffenen Aufsatze, bestehend aus einem hohlen Glasstöpsel mit einem Tropftrichter und einem angeschmolzenen Absorptionsgefüsse, welches, mit passender Flüssigkeit beschickt, die von der entweichenden Luft mitgeführten Halogendämpfe zurückhält.

18) Ein zweiter, ebenfalls mit Hilfe dieses Apparates eventuell in Betracht kommonder Weg besteht darin, zu der die freien Halogene onthaltenden, entsprechend vordünnten flüssigkeit eine Lösung ron Borax im genügenden Überschusso zuzusetzen. Die bei der Umsetzung frei werdende Borsäure dürfte nun nach dem früher Gesagten, ohne dass eine weitere Reduction des Jodations durch das entstehende Jodion zu befürchten
In Anschluss an das Gesagte seien noch kurz die bei der Linwirkung von Bromat bez. Chlorat auf die verschiedenen Ilalogenide bestchenden Verhältmisse in Betracht gezogen.

Was die Einwirkung von Bromat auf die einzelnen Halogenide anbelangt, so unterscheidet sich diese in gewisser Beziehung wesentlich von der des Jodats. Während letzteres bei Gegenwart von Jodid schon bei Zusatz von einem Tropfen einer 1/10 N.Säure eine deutliche Jodausscheidung erkennen lässt, erfolyt bei Anwendung von Bromat und Jodid der Eintritt der Oxydation erst bei Zusatz von einigen cem 1/10 N.-Säure. Die Menge der anzuwendenden Süure, die zur linleitung des Reactionsverlaufes erforderlich ist, ist naturgemäss noch grösser bei einem Gemische von Bromat und Bromid. Wurden z. B. 50 ecm 1/10 N.-K Br $\mathrm{O}_{3}$ und $25 \mathrm{cem} 1 / 10 \mathrm{~N} . \mathrm{KBr}$ verwendet, so mussten ca. $100 \mathrm{ccm} 1 / 10 \mathrm{~N} \cdot-\mathrm{H}_{2} \mathrm{SO}_{4}$ zugegeben werden, bis das Auftreten einer schwach gelben Färbung bei zugefügtem Schwefelkohlenstoff den Eintritt der Reaction anzeigte.

Wird eine Lösung von Bromat mit Jodid und Essigsäure versetzt, so findet schon bei gewöhnlicher Temperatur eine beträchtliche Jodausscheidung statt. Dieselbe ist natürlich bedeutend geringer, wenn neben Essigsüure Natriumacetat in der Lösung vorhanden ist. Bei Bromat, Bromid und Essigsäure findet eine schwache Linwirkung bei gewöhnlicher Temperatur statt, die aber beim Erwärmeı zunimmt. Eine Lösung von Bromat mit überschüssigem Bromid wurde mit Essigsäure und Natriumacetat in genügender Menge versetzt; es fand bei gewöhnlicher Temperatur auch bei mehrstündiger kinwirkung keine Bromausscheidung statt und erst nach ca. zwei Tagen war eine schwache Gelbfärbung zu beobachten. Eine Lösung von Bromat und Chlorid, mit überschüssiger Lssigsäurc versctzt, zeigt bei gewöhnlicher Temperatur eine kaum merkliche Chlorausscheidung; dieselbe tritt aber beim Erwärmen ein.

Die quantitative Untersuchung des Reactionsverlaufes bei der Einwirkung von Bromat auf die Halogenide bei gewöhnlicher Temperatur, in Abhängigkeit von der Concentration, Säuremenge und Zeit, kann erst, ähnlich wie

wäre, die Bestimmung der Halogene, die vielleicht wieder mittels Natriumarsenit vorgenommen werden könnte, gestatten. Voranssetıung bei diesem Verfahren ist, dass die Borsäure in keiner Hinsicht den raschen und guantitativen Vorlauf dor: Judtitration mittels Natriumarsenit in ungünstigen Sinne beeinflusst. Die nothwendigen Versuche bezäglich der Prüfung dieser beiclen $W^{\top}$ ege sowie die evencuelle Anwendung eines resultirenden, brauchbaren Verfahrens für den angegebenen Zweck behalten wir uns vor. 
die Einwirkung von Jodat auf Bromid, nach Auffindung eines hierzu geeigneten Verfahrens durchgeführt werden. Ein solches wird sich vielleicht auf das obon angegebene Verhalten von Bromat gegenüber Bromid und Essigsäure bei Gegenwart einer genügeaden Menge von Natriumacetat, wobei keine Reduction eintritt, basiren lassen. Wenn man nach stattgehabter Reduction des Bromats das freie Brom, ohne dass hierbei eine weitere Reduction des Bromats eintreten kann, ermittein wollte, so könnte man nach vorgenommener Verdünnung wieder bei Anwendung des oben angegebenen Apparates einen genügenden Überschuss von Natriumacetatlösung und hierauf einen solchen von Natriumarsenit zuHliessen lassen; den Überschuss des letzteren könnte man dann entweder, wenn dies möglich, direct in essigsaurer Lösung, sonst nach Abstumpfung der freien Säure, wie gewöbnlich, ermitteln.

Es erübrigt noch, das Verbalten von Chlorat gegenüber den drei Halogeniden in den Kreis unserer Betrachtung zu ziehen.

Wird eine wässerige Lösung von Kaliumchlorat mit Kaliumjodid und ziemlich verdünnter Salzsäure versetzt, so findet momentan fast keine und erst nach längerer Zeit eine ganz schwache Jodausscheidung statt, auf welchen Umstand auch schon. Winkle $r^{19}$ ) hingewiesen hatte. Setzt man aber concentrirte Salzsäure in beträchtlichem Überschusse zu der mit Jodkaljun versetzten Kaliumchloretlösung, so scheidet sich Jod in grosser Menge aus. Der nothwendige Säureüberschuss bis zum Eintritte der Reaction ist bei Chlorat also ein noch bedeutend grösserer als bei Bromat. Über den quantitativen Verlauf der Reaction unter bestimmt eingehaltenen Unständen hat einer von uns (D.) schon gelegentlich der Ausarbeitung einer Methode zur Bestimmung von Chlorat neben Hypochlorit ${ }^{21}$ ), verschiedene Versuche durchgeführt, auf welche hier verwiesen sei. Sowohl die Einwirkung von Chlorat auf überschüssiges Jodid als auch auf Bromid verläuft erst bei sehr grosscm Überschusse an concentrirter Salzsäure bei

19) Dingler, Polyt. Journal 198, 143.

20) Zeitschr. f. angew. Chemie 1899,1195 n. 1217. - Während der Vornahme der Correctur erschien eine Abhandlung von H. Ditz (ChemikerZtg. 1901, 727); in derselben wird die in der ersten Mittheilung (loc. cit.) angegebene Methode zur Untersuchung von Chlorat-Hypochloritgemischen modificirt und das nun vereinfachte Verfahren ausser für diesen Zweek auch zur Bestimmung von Chlorat in den Laugen aus den Absorptionsgefässen bei der Darstellung des Kaliumchlorats empfohlen. In dieser Abhandlung sind noch eine Reihe von Versuchen angegeben bezüglich des Einflusses der Süuremencre, der Concentration und der Zeit bei der Kinwirkung von Kaliumchlorat auf Kaliumbromid und Salzsäure. gewöhnlicher Temperatur vollständig. Der Reactionsverlauf erwies sich bei den früher angestellten Versuchen als abhängig von der Concentration, der Grösse des Säureüberschusses und der Zeit; cin ganz bestimmter Säureüberschuss ist nothwendig. um bei Vorhandensein ron überschüssigem Bromid die quantitative Reduction des Chlorats durchzuführen.

Einige wenige vorläufige Versuche, die zur Beleuchtung des Reactionsverlaufes in dem in der fruber Apparate zurVermeidung von Bromverlusten $\left.{ }^{21}\right)$ durchgeführt wurden, seien hier angeführt.

$25 \mathrm{ccm}$ einer Kaliumchloratlösung, enthaltend $0,075665 \mathrm{~g} \mathrm{KCl} \mathrm{O}_{3}$, verbrauchten bei Anwendung von $10 \mathrm{ccm} 10$ proc. Kaliumbromidlösung und $5 \mathrm{ccm}$ conc. Salzsäure nach vorgenommener Verdünnung und Umsetzung mit Jodkalium bei fünf Minuten langer Linwirkungsdauer des Reductionsmittels $0,1 \mathrm{ccm}$ 1/10 N.- $\mathrm{Na}_{2} \mathrm{~S}_{2} \mathrm{O}_{3}$ für das ausgeschiedene Jod; bei einstündiger linwirkungsdauer $0,45 \mathrm{ccm}$ $1 / 10$ N.- $\mathrm{Na}_{2} \mathrm{~S}_{2} \mathrm{O}_{3}$. Bei Anwendung von $15 \mathrm{ccm}$ conc. $\mathrm{HCl}$ und 5 Minuten langer Einwirkung wurden $9,3 \mathrm{ccm}$, bei einstündiger Einwirkung $15,1 \mathrm{ccm} 1 / 10 \quad$ N.- $\mathrm{Na}_{2} \mathrm{~S}_{2} \mathrm{O}_{3}$ verbraucht.

Bei Anwendung von $20 \mathrm{ccm}$ conc. $\mathrm{HCl}$ betrug der Verbrauch an ${ }^{1 / 10} \mathrm{~N} .-\mathrm{Na}_{2} \mathrm{~S}_{2} \mathrm{O}_{3}$ nach 5 Minuten langer Einwirkung 24,3 ccm, nach einstündiger Einwirkung $32,15 \mathrm{~cm}^{22}$ ).

21) Die hier eingeschlagene Arbeitsweise, bei welcher nach entsprechend vorgenommener Verdunnung, wobei dis mit der entweichenden Luft mitgerissene Brom durch eine Jodkaliumlösung zurückgehalten wird, Jodkaliumlösung zur Umsetzung des Broms zugegeben wird, ist auch bei unvollständigem Verlaufe der Reduction, also bei Vorhandensein von noch unzersetztem Chlorate in der Lösung einwandsfrei, da nach der Verdünnung eine Einwirkung des überschüssigen Chlorats auf das zugesetzte Jodkalium in der kurzen Zeit nicht eintritt.

${ }^{22}$ ) Die Reaction zwischen Chlorat und überschüssigem Bromid (bei gewöhnlicher Temperatur) verläuft also bei steigendem Zusatze von conc. Salzsäure rascher und vollständiger und ist bei genügendem Salzsäureüberschusse nach den in der früheren Nittheilung angegebenenVersuchen quantitativ. Ohne den noch durch\%uführenden weiteren Untersuchumgen vorgreifen zu wollen, glauben wir schon jetzt mit allem Vorbehalte eine theoretische Erklärung hierfür geben zu können. Während der Oxydationsverlauf bei der Einwirkung von Jodat und auch von Bromat auf die Halogenide sich hauptsüchlich als von der Ionisirungstendenz der Ilalogene und der Wasserstoffionenconcentration abhängig erweist, kommt beim Chlorat die Massenwirkung der zugesetzten Salzsäure noch in anderer Hinsicht als bloss betreffs der Wasserstoffionenconcentration in Betracht. Unserer Ansicht nach ist es als wahrscheinlich anzumehmen, dass durch den Zusatz von grösseren Mengen cone. Salzsäure die Dissociation der im Verhältniss zu der letzteren schwächeren Chlorsäure zurückgedrïngt wird und dass die undissociirte Chlorsäure nun die Oxydation herbeiführt. Wird eine genügende Menge conc. Salzsäure zugesetzt, so ist, wie an anderer Stelle mitgetheilte Versuche (Chemiker-Ztg. 1901, 727) ergaben, der 
Wurden zu $25 \mathrm{ccm}$ der $\mathrm{KCl} \mathrm{O}_{3}$-Lösung $10 \mathrm{ccm}$ Wasser (ohne Bromid) und $20 \mathrm{~cm}$ conc. Salzsäure zugesetzt, so wurden bei fünf Minuten langer Einwirkung nach vorgenommener Verdünnung und Umsetzung mit Jodkalium $10,4 \mathrm{ccm}{ }^{1 / 10} \mathrm{~N},-\mathrm{Na}_{2} \mathrm{~S}_{2} \mathrm{O}_{3}$ zur Titration des ausgeschiedenen Jods, also bedeutend weniger als bei der Reduction durch Bromid $(24,3 \mathrm{ccm})$ unter gleichen Cmständen verbraucht. Der zuletzt angegebene Versuch zeigt, dass der Reactionsverlauf bei der Reduction des Chlorats durch Bromid und Salzsäure unter sonst gleichen Umständen rascher verläuft, als bei Anwendung von Salzsäure allein, entsprechend der geringeren Ionisirungstendenz des Broms ${ }^{23}$ ).

Die Reduction des Chlorats durch die Halogenide erfolgt bei gewöhnlicher Temperatur, wie ersichtlich, erst bei Vorhandensein von grossen Mengen überschüssiger Säure; beim Bromat ist der nothwendige Säureüberschuss für den Eintritt bez. den vollständigen Verlauf der Reaction ein bedeutend geringerer als beim Chlorat, wäbrend die Reduction des Jodats durch Jodid vollständig schon bei Anwesenheit der theoretischen Säure- bez. Wasserstoffionenmenge verläuft, die durch Bromid und Chlorid steigende, überschüssige Säuremengen bedarf.

Die genaue Feststellung des Reactionsverlaufes bei der Einwirkung von Bromat und Chlorat auf die Halogenide mit Berücksichtigung der verschiedenen in Betracht kommenden Factoren, sowie die Vervollständigung der angestellten Untersuchung über die Einwirkung von Jodat auf die Halogenide sollen den Gegenstand einer weiteren Arbeit bilden. Wir behalten uns auch vor, die aus der vorstehenden Untersuchung sich ergebenden analytischen Anwendungen wie die Bestimmung von Jodiden neben Bromiden bez. Chloriden etc. durchzuführen.

Brünn, Ende Juli 1901. Chemisch-technologisches Laboratorium der k. k. Technischen Hochschule.

quantitative Reactionsverlauf ein momentaner, was damit erklärt werden könnte, dass die Chlorsäure dann zum grossen Theile im undissociirten Zustande vorhanden ist. Dass sich, die Richtigkeit dieser Ansicht vorausgesetzt, leicht weitere Schlnssfolgerungen über das Wesen der hier je nach den Versuchsbedingungen wechselnden Reactionsgeschwindigkeit ziehen liessen, wollen wir hier nur angedeutet haben.

23) Wird eine Kaliumchlorat und Jodkalium enthaltende Lösung mit überschüssiger 20 proc. Essigsäure versetzt, so bleibt die Lösung bei gewöhnlicher Temperatur Anfangs unveründert, nach einiger Zeit tritt ganz geringe Gelbfärbung auf; diese verstärkt sich beim Erwärmen. Chlorat und Bromid giebt mit Essigsäure bei gewöhnlicher Temperatur keine Bromausscheidung und ist auch beim Erwärmen, nach einem qualitativen Versuche, das Auftreten von Brom nicht zu bemerken.

\section{Eine neue Form von Reagenspapier.}

$\mathrm{V}_{\text {on }}$ Dr. Karl Dieterich, Helfenberg.

Sowohl bei der Prüfung irgend einer Flüssigkeit auf ibre Reaction, als auch bei der Neutralisation saurer oder alkalischer Flüssigkeiten ist der Gebrauch der jetzigen Lakmuspapiere insofern als unpraktisch zu bezeichnen, als man fortgesetzt sowobl rothes wie blaues Lakmuspapier nach einander verwenden muss, was nicht nur einen grossen Verbrauch von Reagenspapier selbst bedeutet, sondern auch zeitraubend ist. Es ist daber wünschenswerth, dass man vermittelst eines Papieres 2 und mehrere Reactionen anstellen kann, bei denen also z. B. sowobl der rothe wie der blaue Lakmusfarbstoff $n$ eben einander auf einem Papier vereinigt sind.

Es ist mir gelungen, ein derartiges Papier, welches rothen und blauen Lakmusfarbstoff in getrennten, haltbaren Schichten neben einander in dünnen Streifen enthält, herzustellen ${ }^{1}$ ) und $\mathbf{z w a r}$ so, dass vermittelst besonderer maschineller Vorrichtungen die Farbstofflösungen in Streifen neben einander anfgestrichen und, um zu verbüten, dass die Säure des rothen Farbstoffes in die blaue durch die Papierfasern übertritt, durch einen isolirenden dünnen Streifen von Ceresin, Parafỉn, überhaupt einen Wasser nicht zugänglichen, indifferenten Körper getrennt sind. Ein auf diese Weise hergestelltes Papier, welches sich natürlich uur auf Schreibpapier, nicht auf Filtrirpapier herstellen lässt, hat den Vortheil, dass man mit einem kleinen Stück sofort 2 Reactionen anstellen kann und auf diese Weise nicht nur Material, sondern auch Zeit spart.

Ebenso, wie man nun blauen und rothen Lakmusfarbstoff neben einander vereinigen kann, kann man auch noch andere Indicatoren, z. B. für die Untersuchung auf Zucker und Eiweiss für die Harnanalyse, weiterhin auch nicht nur 2, sondern 3,4 und mehr Reagentien, wieder getrennt durch diese isolirenden Schichten, auf einem Papier vereinigen, und so mit einem Papier drei, vier und mehr Reactionen auf einmal durch entsprechende Combinationen anstellen.

Der Werth dieser Papiere liegt nicht nur in ihrer praktischen Bedeutung, ihrem sparsamen Gebrauch, sondern auch darin, dass sie durch die isolirenden Schichten dauernd haltbar sind und eine Diffusion der einzelnen Reagentien durch die isolirenden Schichten unmöglich gemacht wird. Diese auf Schreibpapier einseitig gestrichen hergestellten Lakmuspapiere können natürlich nur zum Tüpfeln verwandt werden und zeigen eine Empfindlichkeit, welche den hier in Helfenberg hergestellten Reagenspapieren analog ist.

Die Chemische Fabrik Helfenberg A.-G. vorm. Eugen Dieterich in Helfenberg stellt nach meinem Verfahren vorläufig Lakmuspapier mit rothen und blauen Streifen neben einander her und liefert dasselbe (unter der Benennung "Duplitest") in Bogen von der Grösse 28/44 cm auf Postpapier einseitig gestrichen, und zwar entweder perforirt oder nicht perforirt.

1) D.R.P. No. 123666 . 